\title{
Evaluación de la autoeficacia de emprendimiento y las capacidades de innovación en emprendedores del servicio turístico
}

\section{Evaluation of the self-efficacy of entrepreneurship and innovation capacities in entrepreneurs of the tourism service}

Wendy Diana Carranza Quimi

Universidad Técnica Estatal de Quevedo, Quevedo, Ecuador

wcarranza@uteq.edu.ec

http://orcid.org/0000-0003-4320-7354

Evelyn Janina Carranza Quimi

Investigador Independiente, Quevedo, Ecuador

evelyncarranza14@hotmail.com

http://orcid.org/0000-0002-9706-4769

Luis Oswaldo Manosalvas Vaca

Universidad Estatal Amazónica, Puyo, Ecuador

lmanosalvasvaca@gmail.com

(i) https://orcid.org/0000-0002-4659-8090

Recepción: 16/04/2021 | Aceptación: 19/05/2021 | Publicación: 10/09/2021

Cómo citar (APA, séptima edición):

Carranza Quimi, W. D., Carranza Quimi, E. J, y Manosalvas Vaca, L. O. (2021). Evaluación de la autoeficacia de emprendimiento y las capacidades de innovación en emprendedores del servicio turístico. INNOVA Research Journal, 6(3), 190-210. https://doi.org/10.33890/innova.v6.n3.2021.1743

\section{Resumen}

La investigación analiza el comportamiento emprendedor e innovador de los prestadores de servicios del sector turístico, utilizando como estudio de caso el Cantón Baños de Agua Santa, ubicado en la Provincia de Tungurahua. Se plantearon objetivos que van direccionados a diagnosticar, caracterizar y determinar la situación actual de los emprendedores y su influencia en el entorno que se desempeñan, de la misma manera, identificar si existe una relación entre la autoeficacia emprendedora y las capacidades de innovación. La metodología utilizada tiene un enfoque cuantitativo mediante el uso de instrumentos de medición validados y confiables para 
evaluar la autoeficacia emprendedora y las capacidades de innovación; el diseño de la investigación fue no experimental, con un horizonte transversal, de campo y documental con un análisis descriptivo y correlacional. Los resultados indicaron un perfil demográfico masculino predominante, con una edad joven adulta, y educación máxima alcanzada de bachillerato, la mayoría de estos emprendimientos pertenecen a servicios de alimentos y bebidas, seguido de alojamiento turístico, en donde no se identifica influencia recibida por familiares, también se demostró que los emprendedores no tienen un nivel adecuado de autoeficacia emprendedora y capacidad innovadora lo que limita el desarrollo de la actividad de emprendimiento, sin embargo, las relaciones desarrolladas por las redes con los miembros de la comunidad y la intención de innovar es fuerte al igual que el afrontar cambios inesperados del entorno. Existe una correlación positiva y significativa entre la autoeficacia emprendedora y las capacidades de innovación.

Palabras claves: autoeficacia; capacidad; emprendimiento; innovación turismo.

\begin{abstract}
This research analyzes the enterprising and innovative profile of service providers in the tourism sector, it's used Baños de Agua Santa Canton as a case study, located in Tungurahua Province. Objectives settled, are directed to diagnose, characterize and determine the current situation of entrepreneurs, in the same way, identify if there is a relationship between entrepreneurial selfefficacy and innovation capacities. The methodology used has a quantitative, approach through the use of validated and reliable measurement instruments to evaluate self-efficacy enterprising and innovation capacities; the research design was non-experimental, with horizon cross-sectional, filed and documentary with descriptive analysis. The results indicated a predominant male demographic profile, with young adulthood, and high school education, most of these enterprises belong to food and beverage services, followed by tourist accommodation, where no influence received by relatives is identified. It was also shown that entrepreneurs do not have an adequate level of entrepreneurial self-efficacy and innovative capacity, which limits the development of entrepreneurial activity, however, the relationships developed by the networks with community members and the intention to innovate is strong as well as facing unexpected changes in the environment. There is a positive and significant correlation between entrepreneurial self-efficacy and innovation capacities.
\end{abstract}

Keywords: self-efficacy; capacity; entrepreneurship; innovation; tourism.

\title{
Introducción
}

El emprendimiento es uno de los términos más utilizados en la actualidad, ganando popularidad como alternativa de solución para problemas económicos en los países. El mundo lo ha interpretado como un método de crecimiento para todas las áreas en las cuales se desempeña una persona emprendedora. América Latina ha tenido un crecimiento considerable en su economía, teniendo en cuenta que en circunstancias anteriores se la examinó como un territorio poco emprendedor, su tasa de emprendimiento es muy destacada, aun cuando estos son pequeños, las actividades de los negocios han presentado un gran interés para la generación de cambios significativos respecto a competitividad y desarrollo. El dinamismo emprendedor presenta grandes desafíos que están asociados con el fracaso de los emprendimientos nacientes, su principal objetivo es la satisfacción de sus propias necesidades, sin mirar los posibles obstáculos en los cuales una 
empresa puede estar sujeta. En Ecuador, estas actividades se pueden desarrollar desde una perspectiva turística, ya que el sector presenta gran diligencia y capacidad de crecimiento, de manera que se muestra como una oportunidad para el país en ámbitos económicos (Oyarvide, Nazareno, Roldán, y Ferrales, 2016). Para considerar esta oportunidad, es necesario definir las competencias que deben impulsar los involucrados, en su accionar en varios ámbitos y de igual manera, identificar las aptitudes y actitudes con los clientes. Cubrir una necesidad o un propósito dependerá mucho del contacto y la interacción profesional. El aprendizaje, desarrollo y fortalecimiento de estas habilidades son un factor clave en la organización (Botia y Orozco, 2012). El objetivo de la investigación es analizar el perfil emprendedor mediante la autoeficacia emprendedora y la relación con la capacidad de innovación en emprendedores del sector turístico del cantón Baños de Agua Santa, ubicada en la provincia de Tungurahua del Ecuador, por ser considerada una de las principales localidades para el desarrollo de la actividad turística. El conocimiento de las habilidades emprendedoras y de innovación que responden a las demandas regionales, permitirá contribuir al sector económico de forma adecuada y eficaz.

\section{Problemática}

Baños de Agua Santa es uno de los principales atractivos turísticos en el Ecuador, ubicándose en el puesto número once del ranking del Ministerio de Turismo (MINTUR) en el año 2019, ya que posee una gran variedad de atractivos turísticos, los cuales son de gran relevancia e interés para sus visitantes, promoviendo así la generación de una amplia oferta de emprendimientos para el desarrollo de las actividades en el sector turístico (MINTUR, 2019). Según el catastro nacional del año 2019, emitido por el Ministerio de Turismo, actualmente en el cantón existen aproximadamente 201 establecimientos dedicados a las diferentes actividades turísticas. Considerando el Global Entrepreneurship Monitor GEM, (2018) muchos de los emprendimientos no han mostrado un desarrollo económico notable con el transcurrir del tiempo, ya que se evidencia una deficiencia en la capacidad de generar crecimiento e innovación en los diferentes servicios ofertados (Lasio, Caicedo, Ordeñana, y Izquierdo, 2018).

El emprendedor es aquella persona que toma riesgos para una alta productividad en el mercado, en donde se analizan bajo ciertas perspectivas, entre las cuales tenemos la actitudinal, esta inclina al individuo a responder sobre lo que sucede a su alrededor, dándole la capacidad de actuar, la cual es uno de los principales componentes del perfil emprendedor (Cabana, Cortez, Plaza, Castillo, y Alvarez, 2013). Bajo este contexto, se determinó la necesidad de estudiar el perfil del emprendedor y la capacidad innovadora de los prestadores de servicios turísticos, y así determinar su incidencia como factor que podría impedir el crecimiento de estos establecimientos, por lo tanto, se plantearon las siguientes preguntas de investigación:

¿Los emprendedores de los servicios turísticos de Baños de Agua Santa tienen capacidad innovadora?

¿Cuáles son las características de la autoeficacia emprendedora en los prestadores de servicios turísticos del cantón Baños de Agua Santa?

¿Existe una relación entre la autoeficacia emprendedora y las capacidades de innovación de los prestadores de servicios turísticos del cantón Baños de Agua Santa? 


\section{Marco teórico}

El volumen de investigación relacionada con el emprendimiento en revistas académicas ha aumentado de manera significativa y completa (Volery y Mazzarol, 2015). Los estudios de emprendimiento han tenido gran relevancia en todo tipo de investigaciones, ya que estas ideas corresponden a la creación de algo nuevo. El emprendimiento y sus derivados han influido en todas las industrias y niveles de la sociedad, porque se trata de innovación, competitividad, productividad, generación de riqueza y creación de empleo (Liu y Fang, 2016; Luu, 2017). El término, hace referencia a oportunidades identificadas, en las cuales el emprendedor inicia diferentes actividades que sean viables en la empresa. La intención del emprendedor se produce cuando este organiza sus recursos, entre estos su comportamiento, el cual se ha procurado explicar a partir de diferentes variables investigadas desde otras perspectivas, existiendo vacíos relevantes para el desarrollo económico de los pueblos (Díaz, 2015).

La industria del turismo es un motor fundamental para el crecimiento económico en muchos países, ya que ha superado la tasa de crecimiento de muchas otras industrias incluso dentro de entornos sometidos a turbulencias económicas (Webster y Ivanov, 2014). Pequeñas y medianas empresas de Turismo, en particular, juegan un papel cada vez más destacado en relación con la oferta de los servicios de alojamiento, la creación de empleo, el estímulo económico y la construcción de la imagen y desarrollo equilibrado de los destinos (Hallak et al., 2015). Los mercados de consumo turístico encuentran su lugar en proyectos emprendedores que se caracterizan por sus lugares de acción y los actores que los ejecutan. Los emprendedores buscan nuevos desafíos, centrándose en su comportamiento, para obtener éxito significativo en el mercado (Mota, Dos Anjos y Da Silva, 2017).

El análisis de los emprendedores con la industria turística, se han centrado en características determinadas, con el fin de conocer su intención en el área de emprendimiento. Estas características determinarán sus capacidades en los diferentes ámbitos, entre los cuales tenemos a la innovación y la autoeficacia en emprendimiento (Ubierna, 2015). Por lo tanto, el turismo es considerado una variable netamente económica, ya que abre nuevas perspectivas en un mercado tan versátil y rápido. Sin embargo, la formación de los profesionales en la industria debe ampliarse, a tal punto de formarlos en aspectos multidisciplinarios, es decir con todo el conocimiento necesario para ser un emprendedor en la variedad de servicios a ofrecer (Moreno, 2012).

\section{Emprendimiento en el sector turístico}

El turismo es regido mundialmente por la Organización Mundial del Turismo (OMT), la cual se encarga de esta actividad, promoviendo el desarrollo incluyente y apoyando a la expansión de este movimiento de la mejor manera. Contribuye al sector socioeconómico, con el fin de alcanzar sus objetivos y reducir los posibles impactos negativos. Genera conocimiento de todos aquellos instrumentos que promueven eficazmente la ejecución de los proyectos turísticos (OMT, 2019).

El turismo puede ser estudiado desde diversos aspectos y pautas, debido a la particularidad que presenta frente a las relaciones de los elementos involucrados. Estudios existentes sobre 
emprendimiento turístico han abordado una serie de factores que ilustran la gran variedad y el alcance de las áreas del turismo en las que el espíritu empresarial es relevante (Presenza, Abbate, Meleddu y Sheehan, 2020). Uno de estos factores es el económico y su manera de industrialización en el mundo, esta actividad promueve la transformación a nivel local, involucrando diferentes ámbitos, entre los cuales tenemos al emprendimiento (Sancho et al.,2011). El espíritu empresarial en el turismo ha sido descrito como una estrategia para el desarrollo económico de las regiones, $\mathrm{y}$ varios países apoyan la creación de nuevas empresas, especialmente en las zonas subdesarrolladas (Solvoll et al., 2015). Las iniciativas de emprendimiento en el sector turístico, enfocado a los servicios, procederán de muchas maneras, con el fin de abarcar toda la actividad, permitiendo que los negocios promocionen lo que poseen (Julca, 2011). Teniendo en cuenta que deben ser responsables con los actores involucrados y generar avances en la economía del sector de servicios turísticos, que es donde se desarrollan (Guessa y Toledano, 2011).

Además de la búsqueda de beneficios económicos, el emprendimiento en Turismo se basa en la preservación y evitar la destrucción del medio ambiente natural al evaluar los resultados o consecuencias de la acción empresarial para buscar oportunidades (Shepherd, 2015). En lugar de solo desear maximizar las ganancias, los emprendedores pueden estar motivados en gran medida por el impulso interno de generar una libertad organizacional, por ejemplo, la libertad financiera, para compensar el dilema económico causado por la estacionalidad de trabajo (Solvoll et al., 2015). A pesar del aumento del interés y la singularidad de actividades empresariales en turismo, muy pocos estudios han evaluado el progreso de la investigación sobre emprendimiento e innovación. El emprendimiento es actualmente rico en práctica pero pobre en teoría, por lo que investigadores indican que se debe examinar más a fondo los estudios de emprendimiento en turismo (Shepherd, 2015).

\section{Perfil Emprendedor}

A lo largo de la historia el emprendedor ha sido objeto de estudio en diversas áreas como la economía y la administración, definiéndolo como la persona capaz de generar oportunidades dentro de un negocio, asumiendo riesgos y resolviendo problemas. Estudios previos sugieren la presencia de características existentes que definen a un emprendedor (Alam, Nor, Ali, Omar, y Wel, 2018). Aunque en las investigaciones no existe un acuerdo sobre el perfil de emprendedor, se han identificado rasgos que lo pueden caracterizar como la edad, sexo, condición sociodemográfica o económica (Castro, Scheede, y Zermeño, 2020). El estudio del perfil emprendedor se debe realizar desde dos líneas, como son sus atributos personales y motivacionales, por lo que se ha desarrollado un análisis en base a tres características determinadas como individuales, motivacionales y funcionales (Marulanda, Montoya y Vélez, 2014).

Entre las características personales de un emprendedor se encuentra:

- Adaptabilidad capacidad para incorporar cambios

- Autonomía en la toma de decisiones, libertad de elección

- Confianza en sí mismo tanto en habilidades y capacidades

- Fijación de objetivos y metas desafiantes pero realizables

- Innovación a la apertura e incorporación de nuevas ideas 
- Locus de control interno, concibe que el éxito depende de sí mismo no de factores externos

- Perseverancia constancia sacrificio y entrega

- Poder de persuasión capacidad de influir en los demás para obtener beneficio personal

- Proactividad anticipación a problemas futuros.

- Tolerancia a la incertidumbre capacidad de soportar presión (Marulanda, Montoya y Vélez, 2014).

Otra caracterización que se otorga al emprendedor se da en el sentido de búsqueda de nuevos cambios orientados a la innovación, y a la generación de expectativas y la satisfacción de necesidades, además la incorporación de nuevos y sofisticados conocimientos y tecnologías acorde a las exigencias de la demanda actual. Si bien es cierto, se conoce que el rol del emprendedor es vital en el desarrollo de la economía mundial, definiéndolo como la capacidad de respuesta ante la insatisfacción y aprovechándolo para generar una oportunidad de negocio que satisfaga las necesidades de manera acertada (Julca, 2011). Otro elemento importante son los rasgos de personalidad, los cuales son factores claves para la comprensión de un posible perfil, dentro de los principales se encuentran locus de control, proactividad, riesgo y autoeficacia (Sánchez 2010). El comportamiento emprendedor es un campo de investigación multidisciplinario que analiza características personales, actividades empresariales y aspectos culturales. Hasta la actualidad las características personales, rasgos y actitudes han recibido especial atención para mejorar los perfiles emprendedores, sin embargo, los valores para comprender el comportamiento empresarial han sido más recientes (Esqueda, Csoban, y Prat, 2019).

\section{Autoeficacia emprendedora}

A través del tiempo, la autoeficacia emprendedora de los individuos se ha convertido en el predictor más relevante para comprender la dinámica multifacética detrás de la intención de emprender (Shahab, Chengang, Arbizu, y Haider, 2019). La autoeficacia se define como la capacidad que integra aptitudes internas y externas de la persona, con la finalidad de conseguir algún propósito, el cual es influenciado por la parte emocional del individuo. Este pretende evitar todas aquellas situaciones que excedan su capacidad, y mejorar en aquellas en las cuales se considera bueno, favoreciendo así las actividades que realiza en las diferentes proyecciones de vida. Por lo tanto, las creencias propias del individuo tienen efectos significativos en su trabajo, produciendo cambios y abriendo caminos empresariales (Tsai, Chang, y Peng, 2016).

Según la teoría cognitiva social, la autoeficacia es un mecanismo motivacional que tiene dos funciones para el logro de metas: primero, promueve el desarrollo de metas y, en segundo lugar, fortalece el vínculo entre las metas y el logro de metas. Con respecto a la primera función, la investigación ha proporcionado evidencia consistente de que la autoeficacia empresarial influye positivamente en el desarrollo de los objetivos empresariales. En cuanto a la segunda función, los hallazgos son poco concluyente. De hecho, la evidencia reciente en dominios distintos al emprendimiento sugiere que, durante la lucha por alcanzar la meta, es decir, después de que se hayan formado las metas, la autoeficacia puede obstaculizar el logro de los objetivos (Gielnik, Bledow, y Stark, 2020).). 
La autoeficacia emprendedora, es una herramienta, que recolecta labores importantes de un emprendedor para el manejo de su propia empresa, resaltando las siguientes dimensiones (Guevara, Chávez y Manosalvas, 2018).

- Desarrollar nuevos productos y oportunidades de mercado, las personas de la empresa deben ser dinámicas e inteligentes al momento de desarrollar sus productos, deben considerar la información del cliente y darle seguimiento de acuerdo con su necesidad.

- Construir un entorno innovador, este comportamiento hace referencia a promover un ambiente laboral estable, con personas capaces de generar ideas en beneficio de su entorno

- Iniciar relaciones con inversores, promueve la estabilidad económica mediante la inversión para el desarrollo de la empresa, generando así expansión y beneficio en la misma

- Definir el objeto central del negocio, es necesario tener claro el propósito del negocio o la empresa y todas las áreas que la conforman, con el fin de desempeñar la visión y misión de manera adecuada.

- Afrontar cambios inesperados y desarrollar los recursos humanos clave, se refiere a la habilidad necesaria que tiene el individuo, y que le permite tomar decisiones en situaciones imprevistas, con el fin de saber cómo sobrellevarlas y no entrar en circunstancias poco agradables.

- Desarrollar los recursos humanos clave, el dueño del negocio debe promover en sus trabajadores el cumplimiento de todos sus objetivos, estos individuos deben promover a que la institución se desarrolle de la mejor manera, y la persona indicada para este trabajo es el dirigente de la empresa.

Por lo tanto, al influir en el entorno empresarial, puede comprender otros aspectos y asociarlos de manera positiva (Salvador y Morales, 2009). Empleando iniciativas o propuestas que involucran las siguientes capacidades: marketing, innovación, administración, toma de riesgos y control financiero (Flores, Bojórquez y Ceballos, 2016).

\section{Innovación}

Los estudios de innovación se encuentran determinados como una fuerza dinámica que motiva el desarrollo, adopción y explotación de valor agregado, actividades capaces de transformar las estructuras sociales y económicas (Piñeiro-Chousa, López-Cabarcos, Romero-Castro, y PérezPico, 2020). La innovación es un procedimiento dirigido a los clientes, con el fin de detectar circunstancias positivas y competencias dentro de la organización, que sean de ayuda para promover el interés en los productos y servicios (Alvarez y Bolaños, 2010). Según el Manual Oslo (OECD) (citado de Alvarez y Bolaños, 2010), es la implementacion de algo nuevo para mejorar el producto o servicio, es decir, la utilizacion de métodos significativos que llamen la atención de los clientes, respecto al buen trabajo de la organización. La innovación se considera un proceso complejo e inventivo, cuyos efectos favorecen la economía en general, es por esta razón que gobiernos y entidades promueven su uso en pequeñas y medianas empresas. Durante el proceso se toman grandes decisiones, las cuales están relacionadas al emprendedor, ya que se involucran las capacidades del sujeto al momento de aplicar este procedimiento (Palacio, Arévalo y Guadalupe, 2017). 
Los estudios enfatizan la necesidad de una mayor atención a la investigación sobre la innovación y la transformación en la industria del turismo para aprovechar mejor las oportunidades relacionadas con la demanda de productos basados en la experiencia. La exploración y explotación de tales oportunidades requieren acción empresarial (Presenza, Abbate, Meleddu y Sheehan, 2020). La necesidad de innovar se ha convertido en una condición previa para la supervivencia, sostenibilidad y el futuro crecimiento de las industrias modernas que operan en un entorno altamente competitivo en un mercado global. De los diferentes subsectores del mundo de la economía, el turismo se encuentra entre los más competitivos (Divisekera, y Nguyen, 2018).

El turismo tiene una fuerte necesidad de innovación y las empresas deben responder a la creciente y cambiante demanda turística de una manera innovadora lo cual explica la importancia del espíritu empresarial en turismo (Lebe, et al., 2014). Las empresas establecidas han podido, hasta cierto punto, innovar y desarrollar sus ofertas para satisfacer la creciente demanda de experiencias únicas, creando recuerdos, compromiso y participación emocional para los turistas. Por lo tanto, la industria del turismo depende en gran medida en nuevas empresas tanto para servir al crecimiento del mercado como para apoyar la innovación (Rodríguez-Sánchez, Williams, y Brotons, 2019).

\section{Capacidad de Innovación}

Los emprendedores señalan a la innovación como fuente prioritaria para conseguir éxito, ya que dentro de un mercado competitivo la innovación es una herramienta relevante, elevando el potencial de la organización y generando un impacto económico a largo plazo, por lo que los emprendedores pretenden que la innovación sea medible y controlable siendo necesario el desarrollo de capacidades y habilidades, buscando así que la capacidad de innovación sea la llamada a desempeñar esta labor La capacidad de innovación se puede describir como la capacidad de transformar continuamente el conocimiento y las ideas en nuevos productos, procesos y sistemas en beneficio de la empresa y sus partes interesadas. La capacidad de innovación no solo se refiere a la capacidad de tener éxito en la gestión de una nueva corriente empresarial, también le interesa la capacidad de sintetizar paradigmas operativos (Omar, Aris, y Nazri, 2016).

Son pocos los estudios que vinculan la capacidad innovadora dentro del sector de servicios ya que la mayor parte de estos se han enfocados en industrias y tecnología, sin embargo, resulta erróneo generalizar esta definición ya que afronta diversos retos al momento de la identificación de capacidades, características, habilidades para el desarrollo acertado de la gestión de innovación. Originalmente la capacidad innovadora se abordaba con una óptica tecnológica, sin embargo, abarca aspectos como producción, aprendizaje, asignación de recursos, mercadeo, organización, entre otras, las cuales ayudan a una mejor práctica de lo aprendido. Las firmas establecidas y las firmas más grandes han podido, hasta cierto punto, innovar y desarrollar sus ofertas para satisfacer la creciente demanda de experiencias únicas, creando recuerdos y compromiso y participación emocional para los turistas. Por lo tanto, la industria del turismo depende en gran medida en nuevas empresas tanto para servir al crecimiento del mercado como para apoyar la innovación (RodríguezSánchez, Williams, y Brotons, 2019) 
Hogan et al, 2011 (citado de García, Quintero y Arias, 2014) proponen tres dimensiones de capacidad innovadora, dentro de las que se encuentran:

- Capacidad de innovacion orientadas hacia el cliente: Habilidad para generar nuevos productos y servicios y resolución de inconvenientes y dificultades de forma innovadora

- Capacidad de innovación orientada al mercado: Implementación de programas innovadores que permiten conocer al merccado generando asi productos y servicios que vayan a la par con la necesidad del cliente.

- Capacidad de innovación orientada a la tecnología: Adaptación de nuevas y modernas tecnologías que sobresalgan de los competidores (García, Quintero y Arias, 2014).

Según los autores Selznick y Mayhew (2018) indicaron que las dimensiones relevantes del constructo Capacidad de innovación son:

- Motivación, conjunto de causas por las que las personas actúan frente a diferentes circunstancias.

- Proactividad, una persona proactiva decide sobre su propia vida, se centra en las cosas que puede hacer asumiendo su responsabilidad en las diferentes situaciones.

- Autoconcepto de innovación, hace referencia a lo que cada persona piensa y siente sobre sí mismo en temas de innovación.

- Redes, son procedimientos no planificados, ya que tienden a ser esporádicos y autoorganizados, crean lazos en sus diferentes áreas.

- Comunicación persuasiva, esta dimensión tiene como objetivo conseguir el apoyo del grupo con el fin de llevar a cabo las actividades de una empresa.

- Trabajo en equipo a través de la diferencia, se considera una ventaja ya que genera cambios en la manera de trabajar aumentando la innovación y satisfacción en el trabajo.

- Cognición creativa, esta dimensión se centra en el control del medio interno, es decir la parte consciente de una persona, permitiéndole ejecutar diferentes acciones, en este caso la creativa.

- Intención de innovar, hace una referencia a una dirección consciente hacia un objeto innovador, produciendo un notable cambio en los rasgos de una persona.

- Toma de riesgos/tolerancia, hace referencia a involucrarse en situaciones imprevistas, pueden traer consecuencias o podrían provocar estabilidad económica en la empresa.

\section{Materiales y Métodos}

La investigación tiene un enfoque cuantitativo, descriptivo y transversal. La ruta cuantitativa sigue un paradigma deductivo positivista, que pretende la resolución de preguntas planteadas mediante la medición de la realidad, con frecuencia aplica la estadística y busca ser lo más preciso con la información que se recolecta y analiza. El análisis descriptivo fue necesario ya que permitió identificar las características y los perfiles de los emprendedores (Hernandez, Fernandez y Baptista, 2014). Se realizó una investigación de campo, en donde introduce al individuo en el ambiente, buscando contribuciones adicionales al tema, con el fin de que el estudio 
sea efectivo al momento de presentar resultados El contacto que se tuvo con los emprendedores fue directo, recolectando así la información necesaria para determinar las variables principales planteadas utilizando instrumentos de medición validados sobre autoeficacia emprendedora y capacidad de innovación, previo a una instrucción breve y completa acerca de las encuestas obteniendo un alto grado de respuestas (Hernandez et al., 2014).

La muestra se obtuvo del catastro de los establecimientos turísticos registrados en el Ministerio de Turismo hasta el mes de febrero de 2020, se desarrolló mediante un muestreo estratificado obteniendo una muestra representativa de 201 emprendedores registrados. Se aplicó la herramienta de Autoeficacia Emprendedora de De.Noble et al. (1999), en su versión validada en España por Moriano et al. (2006), la cual mide la perspectiva del emprendedor mediante sus creencias con respecto a sus propias habilidades para realizar diferentes actividades necesarias para un emprendimiento. El número de preguntas planteadas fue de 23, con seis dimensiones por analizar con una escala, que va desde uno (Completamente incapaz) a cinco (Perfectamente capaz). Por otro lado, se aplicó la herramienta Capacidad de Innovación validada por Selznick y Mayhew (2018), esta mide la noción del emprendedor respecto a tres constructos, los cuales se enfocan en las dimensiones intrapersonal, social y cognitiva del individuo. El número de preguntas planteadas fue de 42 y nueve dimensiones por analizar, en una escala Likert, que va desde uno (Totalmente en desacuerdo) a cinco (Totalmente de acuerdo). Los análisis de resultados se realizaron mediante el uso del software estadístico SPSS (Paquete Estadístico para las Ciencias Sociales) versión 24, se desarrolló estadística descriptiva y correlación de Spearman con evaluación previa de normalidad de datos.

\section{Resultados}

\section{Análisis de Confiabilidad}

Un instrumento de medición se considera confiable cuando el valor obtenido es igual o mayor a 0,7 en una escala de 0 a 1 . La consistencia interna de este instrumento se basa en una escala de opciones múltiples (escala Likert), y puede ser analizada, debido a los formatos de calificación y respuesta mediante el Alfa de Cronbach (Quero, 2010). Los instrumentos indicaron valores de 0,88 en autoeficacia emprendedora y 0,95 en capacidad de innovación, por lo tanto, se evidencia una alta confiabilidad.

\section{Tabla 1}

Alfa de Cronbach de instrumentos de medición

\begin{tabular}{ccc}
\hline Variable & Alfa de Cronbach & N de elementos \\
\hline Autoeficacia emprendedora &, 888 & 23 \\
Capacidad innovadora &, 953 & 42 \\
\hline
\end{tabular}




\section{Resultados demográficos}

\section{Tabla 2}

Género de la muestra

\begin{tabular}{llcccc}
\hline & & Frecuencia & Porcentaje & Porcentaje válido & Porcentaje acumulado \\
\hline Válido & Femenino & 84 & 41,8 & 41,8 & 41,8 \\
& Masculino & 117 & 58,2 & 58,2 & 100,0 \\
& Total & 201 & 100,0 & 100,0 & \\
\hline
\end{tabular}

En la tabla 2 se indica un mayor porcentaje del género masculino 58\% en comparación con el género femenino $42 \%$, sin embargo, esto no refleja un aspecto superior en el comportamiento emprendedor e innovador en la muestra analizada.

\section{Tabla 3}

Edad de la muestra

\begin{tabular}{llcccc}
\hline & Frecuencia & Porcentaje & Porcentaje válido & $\begin{array}{c}\text { Porcentaje } \\
\text { acumulado }\end{array}$ \\
\hline Válido & $\mathbf{1 8 - 2 5}$ & 16 & 8,0 & 8,0 & 8,0 \\
& Más de 55 años & 23 & 11,4 & 11,4 & 19,4 \\
& $\mathbf{4 6 - 5 5}$ & 38 & 18,9 & 18,9 & 38,3 \\
& $\mathbf{3 6 - 4 5}$ & 57 & 28,4 & 28,4 & 66,7 \\
& $\mathbf{2 6 - 3 5}$ & 67 & 33,3 & 33,3 & 100,0 \\
& Total & 201 & 100,0 & 100,0 & \\
\hline
\end{tabular}

La tabla 3, determina aproximadamente un $67 \%$ de la muestra en una edad comprendida entre 18 a 45 años de edad, lo cual representa un segmento adulto joven.

\section{Tabla 4}

Estado civil de los emprendedores

\begin{tabular}{llrrrr}
\hline & Frecuencia & Porcentaje & Porcentaje válido & Porcentaje acumulado \\
\hline Válido & Unión Libre & 8 & 4,0 & 4,0 & 4,0 \\
& Viudo & 8 & 4,0 & 4,0 & 8,0 \\
& Divorciado & 18 & 9,0 & 9,0 & 16,9 \\
& Soltero & 55 & 27,4 & 27,4 & 44,3 \\
\hline
\end{tabular}


Evaluación de la autoeficacia de emprendimiento y las capacidades de innovación en emprendedores del servicio turístico

\begin{tabular}{lrrrr}
\hline & Frecuencia & Porcentaje & Porcentaje válido & Porcentaje acumulado \\
\hline Casado & 112 & 55,7 & 55,7 & 100,0 \\
Total & 201 & 100,0 & 100,0 & \\
\hline
\end{tabular}

La tabla 4 señala que el $56 \%$ de la muestra se encuentra con un estado civil en relación de dependencia (casados) y un $27 \%$ son solteros.

\section{Tabla 5}

Nivel de educación

\begin{tabular}{cccccc}
\hline & Frecuencia & Porcentaje & Porcentaje válido & Porcentaje acumulado \\
\hline Válido & Cuarto nivel & 3 & 1,5 & 1,5 & 1,5 \\
& Primaria & 8 & 4,0 & 4,0 & 5,5 \\
& Tercer nivel & 72 & 35,8 & 35,8 & 41,3 \\
& Bachillerato & 118 & 58,7 & 58,7 & 100,0 \\
& Total & 201 & 100,0 & 100,0 & \\
\hline
\end{tabular}

La tabla 5 indica que los emprendedores se encuentran en un $59 \%$ con un nivel de educación de bachillerato y un $36 \%$ en tercer nivel, esto sugiere que no existe una influencia en la educación para ser emprendedor.

\section{Tabla 6}

Tipo de servicio ofertado

\begin{tabular}{ccccc}
\hline & Frecuencia & Porcentaje & Porcentaje válido & Porcentaje acumulado \\
\hline Transporte Turístico & 2 & 1,0 & 1,0 & 1,0 \\
Parques de atracción & 2 & 1,0 & 1,0 & 2,0 \\
Operación e Intermediación & 30 & 14,9 & 14,9 & 16,9 \\
Alojamiento & 70 & 34,8 & 34,8 & 51,7 \\
Alimentos y Bebidas & 97 & 48,3 & 48,3 & 100,0 \\
Total & 201 & 100,0 & 100,0 & \\
\hline
\end{tabular}

La tabla 6 identifica el tipo de servicio turístico ofertado, considerando el segmento de alimentos y bebidas en primer lugar $48 \%$, seguido por el de alojamiento $35 \%$. 


\section{Tabla 7}

Ingresos mensuales

\begin{tabular}{cccccc}
\hline & Frecuencia & Porcentaje & Porcentaje válido & Porcentaje acumulado \\
\hline Válido & Mayor a 2001 \$ & 11 & 5,5 & 5,5 & 5,5 \\
& Menos de 500 \$ & 12 & 6,0 & 6,0 & 11,4 \\
& $\mathbf{1 5 0 1}$ a 2000 \$ & 32 & 15,9 & 15,9 & 27,4 \\
& $\mathbf{1 0 0 1}$ a 1500 \$ & 69 & 34,3 & 34,3 & 61,7 \\
& $\mathbf{5 0 1}$ a 1000 \$ & 77 & 38,3 & 38,3 & 100,0 \\
& Total & 201 & 100,0 & 100,0 & \\
\hline
\end{tabular}

La tabla 7 señala que más del $70 \%$ de la muestra tiene un ingreso mensual entre 500 a 1000 USD.

\section{Tabla 8}

Procedencia del emprendedor

\begin{tabular}{cccccc}
\hline & & & & \\
& & Frecuencia & Porcentaje & Porcentaje válido & Porcentaje acumulado \\
\hline Válido & Extranjero & 10 & 5,0 & 5,0 & 5,0 \\
& Nacional & 191 & 95,0 & 95,0 & 100,0 \\
& Total & 201 & 100,0 & 100,0 & \\
\hline
\end{tabular}

La tabla 8 determina que el mayor porcentaje 95\% de los emprendedores son de procedencia nacional y apenas un $5 \%$ son extranjeros.

\section{Tabla 9}

Contacto con centro u organismo de apoyo al emprendimiento

\begin{tabular}{cccccc}
\hline & & Frecuencia & Porcentaje & Porcentaje válido & Porcentaje acumulado \\
\hline Válido & Si & 62 & 30,8 & 30,8 & 30,8 \\
& No & 139 & 69,2 & 69,2 & 100,0 \\
& Total & 201 & 100,0 & 100,0 & \\
\hline
\end{tabular}

En la tabla 9, se analiza si los emprendedores tuvieron algún contacto con un centro u organismo de apoyo al emprendimiento, los resultados indicaron que aproximadamente el $70 \%$ no tiene relación. 
Evaluación de la autoeficacia de emprendimiento y las capacidades de innovación en emprendedores del servicio turístico

\section{Tabla 10}

Nivel de estudio alcanzado por el padre

\begin{tabular}{cccccc}
\hline & & Frecuencia & Porcentaje & Porcentaje válido & Porcentaje acumulado \\
\hline Válido & Cuarto nivel & 2 & 1,0 & 1,0 & 1,0 \\
& Tercer nivel & 28 & 13,9 & 13,9 & 14,9 \\
& Primaria & 68 & 33,8 & 33,8 & 48,8 \\
& Bachillerato & 103 & 51,2 & 51,2 & 100,0 \\
& Total & 201 & 100,0 & 100,0 & \\
\hline
\end{tabular}

La tabla 10 señala la educación alcanzada por el padre, los resultados indican que el $84 \%$ obtuvo un nivel de estudio primario y bachillerato, lo cual se podría considerar que no influye en el comportamiento emprendedor de los hijos, aunque estaría sujeto a estudio.

\section{Tabla 11}

Nivel de estudio alcanzado por la madre

\begin{tabular}{cccccc}
\hline & Frecuencia & Porcentaje & Porcentaje válido & Porcentaje acumulado \\
\hline Válido & Cuarto nivel & 3 & 1,5 & 1,5 & 1,5 \\
& Tercer nivel & 22 & 10,9 & 10,9 & 12,4 \\
& Primaria & 70 & 34,8 & 34,8 & 47,3 \\
& Bachillerato & 106 & 52,7 & 52,7 & 100,0 \\
& Total & 201 & 100,0 & 100,0 & \\
\hline
\end{tabular}

La tabla 11 señala la educación alcanzada por la madre, los resultados indican que el $87 \%$ obtuvo un nivel de estudio primario y bachillerato, lo cual se podría considerar que no influye en el comportamiento emprendedor de los hijos, aunque estaría sujeto a estudio.

\section{Tabla 12}

Miembro de la familia que posee un negocio o emprendimiento

\begin{tabular}{cccccc}
\hline & & Frecuencia & Porcentaje & Porcentaje válido & Porcentaje acumulado \\
\hline Válido & Si & 82 & 40,8 & 40,8 & 40,8 \\
& No & 119 & 59,2 & 59,2 & 100,0 \\
& Total & 201 & 100,0 & 100,0 & \\
\hline
\end{tabular}


En la tabla 12 se evalúa si algún miembro de la familia posee un negocio o emprendimiento el $59 \%$ indica que no, sin embargo, el $49 \%$ señala que si, esto podría sugerir cierta influencia a tener emprendimientos.

\section{Tabla 13}

Tiempo de apertura del negocio

\begin{tabular}{cccccc}
\hline & Frecuencia & Porcentaje & Porcentaje válido & Porcentaje acumulado \\
\hline Válido & Menor a 1 año & 17 & 8,5 & 8,5 & 8,5 \\
& Entre 5 a 10 años & 44 & 21,9 & 21,9 & 30,3 \\
& Mayor de 10 años & 50 & 24,9 & 24,9 & 55,2 \\
& Entre 1 a 5 años & 90 & 44,8 & 44,8 & 100,0 \\
& Total & 201 & 100,0 & 100,0 & \\
\hline
\end{tabular}

La tabla 13 determina el tiempo de apertura de los negocios, en donde se indica que el 65\% tiene un período que va de 1 a 10 años, por lo cual se manifiesta una relativa estabilidad.

\section{Análisis descriptivo de la variable Autoeficacia Emprendedora}

Con el objetivo de mejorar la interpretación, los instrumentos Autoeficacia Emprendedora y Capacidades de Innovación fueron analizados mediante un proceso de baremo estadístico para hacer una reagrupación de los datos obtenidos en escala de Likert de cinco resultados hacia tres nuevas categorías: débil, medio, fuerte.

\section{Tabla 14}

\section{Análisis autoeficacia emprendedora}

\begin{tabular}{|c|c|c|}
\hline Dimensión & Resultado de Análisis & Interpretación \\
\hline $\begin{array}{l}\text { Afrontar cambios } \\
\text { inesperados }\end{array}$ & $\begin{array}{l}\text { Más del } 76 \% \text { de medio a } \\
\text { fuerte }\end{array}$ & $\begin{array}{l}\text { Indica una actitud positiva del emprendedor hacia } \\
\text { los cambios del entorno. }\end{array}$ \\
\hline $\begin{array}{l}\text { Construir un entorno } \\
\text { innovador }\end{array}$ & Más del $78 \%$ de medio a débil & $\begin{array}{l}\text { Existe limitación en el conjunto de } \\
\text { circunstancias, factores sociales y culturales que } \\
\text { determinan la actividad innovadora en el } \\
\text { emprendimiento. }\end{array}$ \\
\hline $\begin{array}{l}\text { Definir el objetivo } \\
\text { central del negocio }\end{array}$ & Más del $75 \%$ de medio a débil & $\begin{array}{l}\text { Existe la necesidad de enfocar el giro del negocio } \\
\text { para mantener la sostenibilidad del } \\
\text { emprendimiento. }\end{array}$ \\
\hline $\begin{array}{l}\text { Desarrollar los recursos } \\
\text { humanos clave }\end{array}$ & Más del $77 \%$ de medio a débil & $\begin{array}{l}\text { Es necesario conocer las habilidades y } \\
\text { competencias del talento humano y generar } \\
\text { estrategias para el desarrollo en la organización } \\
\text { empresarial turística., }\end{array}$ \\
\hline $\begin{array}{l}\text { Descubrir nuevos } \\
\text { productos y }\end{array}$ & Más del $85 \%$ de medio a débil & $\begin{array}{l}\text { Se evidencia limitaciones de habilidades para } \\
\text { identificar nuevos productos y oportunidades que }\end{array}$ \\
\hline
\end{tabular}


Evaluación de la autoeficacia de emprendimiento y las capacidades de innovación en emprendedores del servicio turístico

\begin{tabular}{cll}
\hline Dimensión & Resultado de Análisis & \multicolumn{1}{c}{ Interpretación } \\
\hline $\begin{array}{c}\text { oportunidades de } \\
\text { mercado }\end{array}$ & & $\begin{array}{l}\text { le permitan al emprendedor potencializar su } \\
\text { negocio. }\end{array}$ \\
$\begin{array}{c}\text { Iniciar relaciones con } \\
\text { inversores }\end{array}$ & Más del $79 \%$ de medio a débil & $\begin{array}{l}\text { No se ha logrado la construcción de alianzas con } \\
\text { potenciales socios e inversionistas. }\end{array}$ \\
\hline
\end{tabular}

\section{Análisis descriptivo de la variable Capacidad de Innovación}

\section{Tabla 15}

\section{Análisis capacidad innovación}

\begin{tabular}{|c|c|c|}
\hline Dimensión & Resultado de Análisis & Interpretación \\
\hline Motivación & Más del $75 \%$ de medio a débil & $\begin{array}{l}\text { La falta de motivación podría influir en la } \\
\text { capacidad innovadora, por ser un estado propio del } \\
\text { individuo, que dirige y mantiene la conducta de la } \\
\text { persona hacia metas o fines determinados }\end{array}$ \\
\hline Proactividad & Más del $72 \%$ de medio a débil & $\begin{array}{l}\text { Los emprendedores del sector turístico deben } \\
\text { desarrollar la actitud para asumir el pleno control } \\
\text { de su conducta de modo activo, lo que implica la } \\
\text { toma de iniciativa en la generación de acciones } \\
\text { creativas y audaces para el negocio. }\end{array}$ \\
\hline $\begin{array}{l}\text { Autoconcepto de } \\
\text { innovación }\end{array}$ & Más del $71 \%$ de medio a débil & $\begin{array}{l}\text { Se evidencia una limitada opinión o descripción } \\
\text { que tiene el emprendedor de sí mismo con respecto } \\
\text { a su capacidad de innovar. }\end{array}$ \\
\hline Redes & $\begin{array}{c}\text { Más del } 77 \% \text { de medio a } \\
\text { fuerte }\end{array}$ & $\begin{array}{l}\text { Los emprendedores se unen por relaciones que } \\
\text { hacen surgir a los grupos con intereses comunes en } \\
\text { los cuales se ven influidos de una forma u otra por } \\
\text { el resto de su comunidad }\end{array}$ \\
\hline $\begin{array}{l}\text { Comunicación } \\
\text { persuasiva }\end{array}$ & Más del $70 \%$ de medio a débil & $\begin{array}{l}\text { Los emprendedores del sector turístico tienen la } \\
\text { habilidad de transmitir estímulos de comunicación } \\
\text { para modificar la conducta de otros individuos en } \\
\text { su entorno. }\end{array}$ \\
\hline $\begin{array}{l}\text { Trabajo en equipo a } \\
\text { través de la diferencia }\end{array}$ & Más del $79 \%$ de medio a débil & $\begin{array}{l}\text { Falta de cooperación para lograr un fin común en } \\
\text { la ejecución de negocios en beneficio para la } \\
\text { comunidad. }\end{array}$ \\
\hline Cognición creativa & Más del $72 \%$ de medio a débil & $\begin{array}{l}\text { No se identifican las estructuras representacionales } \\
\text { y los procesos cognitivos que dan origen a } \\
\text { productos creativos en los emprendimientos. }\end{array}$ \\
\hline Intención de innovar & $\begin{array}{l}\text { Más del } 80 \% \text { de medio a } \\
\text { fuerte }\end{array}$ & $\begin{array}{l}\text { Se evidencia un fuerte propósito para la creación o } \\
\text { modificación de nuevos productos y servicios } \\
\text { turísticos. }\end{array}$ \\
\hline $\begin{array}{c}\text { Toma de } \\
\text { riesgos/tolerancia }\end{array}$ & Más del $77 \%$ de medio a débil & $\begin{array}{l}\text { No se identifica la ocurrencia para desarrollar la } \\
\text { probabilidad de un suceso y sus consecuencias, } \\
\text { mediante el respeto íntegro hacia las ideas, } \\
\text { prácticas o creencias }\end{array}$ \\
\hline
\end{tabular}




\section{Tabla 15}

Análisis de correlación entre Autoeficacia Emprendedora y Capacidad de Innovación.

\begin{tabular}{llcc}
\hline & & Autoeficacia emprendedora & Capacidad de Innovación \\
\hline \multirow{4}{*}{ Autoeficacia emprendedora } & Correlación de Pearson & 1 &, $680^{* *}$ \\
& Sig. (bilateral) & &, 000 \\
& $\mathrm{~N}$ & 201 & 201 \\
& Correlación de Pearson &, $680^{* *}$ & 1 \\
& Capacidad de Innovación &, 000 & \\
& Sig. (bilateral) & 201 & 201 \\
\hline
\end{tabular}

**. La correlación es significativa al nivel 0,01 (bilateral).

Se determina una valoración significativa de $0,680^{* *}$ a un nivel de 0,01 , entre las variables lo cual se establece como una buena correlación (Larico y Montoya, 2019).

\section{Conclusiones}

Con respecto al perfil demográfico se concluye que el género que predomina en el desarrollo de emprendimientos es el masculino, sin embargo, el género femenino tiene un papel relevante en la actividad emprendedora. El rango de edad indica que existe una población emprendedora joven adulta. El estado civil demostró una relación de dependencia con una carga familiar. Los estudios alcanzados por los emprendedores en su mayoría han culminado el nivel bachillerato, sin embargo, existe un porcentaje considerable con títulos de tercer nivel. Los padres de los emprendedores tienen un nivel secundario de educación y no tienen un miembro de la familia que sea emprendedor o dueño de negocio, con lo que se puede inferir que no existe influencia familiar para desarrollar emprendimientos, aunque esto se debe ampliar en investigaciones para identificar otros posibles factores. Los emprendedores en su totalidad provienen de Ecuador y son nativos de la provincia de Tungurahua. Los emprendimientos turísticos generados en su mayoría son de alimentos y bebidas, seguido de alojamiento, debido a que Baños de Agua Santa es una ciudad turística reconocida a nivel nacional e internacional, a pesar de que los encuestados consideran que el apoyo a emprendedores, por parte de los organismos gubernamentales, no ha sido el esperado ya sea por carencia de tiempo, poca difusión y por las temáticas que no han sido de su interés o no van de acorde a sus necesidades. Por otro lado, las ganancias mensuales de los negocios están en un rango de 1000 a 1500 dólares después de cubrir todos sus gastos, y su estabilidad se encuentra alrededor de 1 a 10 años en el mercado.

El análisis de la autoeficacia emprendedora, indicó que todas las dimensiones a excepción de "afrontar cambios inesperados" se encuentran en una categoría media a débil, por lo se puede concluir que existe un limitado conocimiento de las habilidades propias del emprendedor turístico, sin embargo, presentan una actitud positiva hacia los cambios del entorno. La capacidad de innovación que mide la noción del emprendedor con respecto a las actitudes que integran la habilidad para crear innovación, determinó que las dimensiones "redes" e "intención de innovar" son las más fuertes como capacidades de innovación, es decir los emprendedores se unen por relaciones que hacen surgir a los grupos con intereses comunes, evidenciando un fuerte propósito 
para la creación o modificación de nuevos productos y servicios turísticos, sin embargo, las dimensiones "motivación", "autoconcepto de innovación "proactividad", "comunicación persuasiva", "trabajo en equipo a través de la diferencia", "cognición creativa", y "toma de riesgos/tolerancia”, están en una categoría media a débil lo cual da lugar a futuros estudios para comprender el comportamiento de los emprendedores.

Existe una relación significativa entre la Autoeficacia Emprendedora y las Capacidades de Innovación en los emprendedores del sector turístico, por lo tanto, el conocimiento de las propias habilidades de emprendimiento puede mejorar las capacidades de innovación, siempre y cuando se desarrollen las dimensiones analizadas en la investigación, de la misma manera, futuras investigaciones podrían evaluar el efecto causal de la relación de variables.

\section{Referencias bibliográficas}

Alam, SS, Nor, NGM, Ali, MH, Omar, NA., \& Wel, CAC (2018), "Relationship between entrepreneur's traits and cloud computing adoption among malay-owned SMEs in Malaysia". Cuadernos de Gestión, 8(2), 115-131. http://doi.org/10.5295/cdg.140515ss

Alvarez , L., y Bolaños, G. (2010). Innovación y Estrategia: dos conceptos aparentemente contradictorios. Revista Electrónica Nova Scientia, 3(1), 118-142. http://doi.org/10.21640/ns.v3i5.203

Botia, M., y Orozco, L. (2012). Competencias en servicio al cliente y efectividad en solución de problemas: Sus características en el sector turismo. Estudios y Perspectivas en turismo, 21(3), 646-662.

Cabana, R., Cortez, I., Plaza, D., Castillo, M., y Alvarez, A. (2013). Análisis de Las Capacidades Emprendedoras Potenciales y Efectivas en Alumnos de Centros de Educación Superior. Journal of technology management \& innovation, 8(1), 65-75.

Castro, M. P., Scheede, C. R., \& Zermeño, M. G. (2020, April). Entrepreneur profile and entrepreneurship skills: Expert's analysis in the Mexican entrepreneurial ecosystem. In 2020 International Conference on Technology and Entrepreneurship-Virtual (ICTE-V). IEEE.

Esqueda, S., Csoban, E., \& Prat, J. F. (2019). A profile of the popular entrepreneur: a value-based approach. Academia Revista Latinoamericana de Administración. 32(2), 267-281.

Flores, A., Bojórquez, A., y Ceballos, S. (2016). El impacto de la autoeficacia emprendedora en los resultados de innovación de los. Red Internacional de Investigadores en Competitividad, 559-573. https://riico.net/index.php/riico/article/viewFile/1334/1003

García, O., Quintero , J., y Arias, J. (2014). Capacidades de innovación, desempeño innovador y desempeño organizacional en empresas del sector servicios. Scielo, 27(49), 87-108. https://bit.ly/3BrHff6

Gielnik, M. M., Bledow, R., \& Stark, M. S. (2020). A dynamic account of self-efficacy in entrepreneurship. Journal of Applied Psychology, 105(5), 487.

Guessa, A., y Toledano, N. (2011). Turismo , emprendimiento y sostenibilidad en los espacios naturales protegidos.El caso de Andalucía,España. Estudios y perspectivas del turismo, 20, 1154-1174. https://www.redalyc.org/pdf/1807/180722704011.pdf 
Guevara, D., Chávez, G., y Manosalvas, L. (2018). Influencia de las emociones en las habilidades emprendedoras de los propietarios de servicios turísticos de la ciudad de Puyo. [Tesis de grado, Universidad Estatal Amazónica: Puyo, Pastaza, Ecuador]

Hallak, R., Assaker, G., Lee, C., 2015. Tourism entrepreneurship performance: the effects of place identity, self-efficacy, and gender. Journal of Travel Research 54 (1), 36- 51.

Hernandez Sampieri, R., Fernandez Collado, C., y Baptista Lucio, P. (2014). Metodología de la investigación. https://bit.ly/2WDEbNS

Julca Meza, E. (2012). Evaluación de factores básicos de competencia de emprendimiento empresarial en los estudiantes de turismo: El caso de la Universidad San Martín de Porres [Tesis Doctoral, Universidad San Martín de Porres Lima, Perú].

Dias, J. (2015). Instituciones y percepciones institucionales como antecedentes del emprendimiento en economías en desarrollo: una aplicación al sector turístico [Tesis Doctoral, Universidad de las Palmas de Gran Canaria: Las Palmas de Gran Canaria, España].

Divisekera, S., \& Nguyen, V. K. (2018). Determinants of innovation in tourism evidence from Australia. Tourism Management, 67, 157-167.

Larico, D. S., y Montoya, E. E. C. (2019). Competencias gerenciales de los empresarios y su relación con los factores que limitan el crecimiento empresarial de las MYPES agroexportadoras de aceituna y derivados de Tacna. Revista Veritas et scientia-upt, $8(2)$, 1260-1271. https://doi.org/10.47796/ves.v8i2.160

Lasio, V., Caicedo, G., Ordeñana, X., \& Izquierdo, E. (2018). Global Entrepreurship Monitor. Guayaquil, Ecuador: ESPAE ESPOL.

Lebe, SS, Mulej, M., Crnogaj, K., Rebernik, M., Hojnik, BB y Gomezelj, DO (2014). Building a model of researching the sustainable entrepreneurship in the tourism sector. Kybernetes, 43 (3/4), 377-393.

Liu, C.H.S., Fang, Y.P., (2016). Night markets: entrepreneurship and achieving competitive advantage. Int. J. Contemp. Hosp. Manage. 28 (11), 2374-2398.

Luu, T.T., (2017). CSR and organizational citizenship behavior for the environment in hotel industry: the moderating roles of corporate entrepreneurship and employee attachment style. Int. J. Contemp. Hosp. Manage. 29 (11), 2867-2900.

Marulanda, F., Montoya, I., y Vélez , J. (2014). Aportes teóricos y empíricos al estudio del emprendedor. Cuadernos de Administración, $\quad$ 51(30), 89-99. https://www.redalyc.org/pdf/2250/225031330010.pdf

MINTUR (2019). Ministerio de Turismo. https://www.turismo.gob.ec/wpcontent/uploads/2019/11/Fomento-al-emprendimiento-tur\%C3\%ADstico-octubre-2019OK.pdf

Mota, G., Dos Anjos, F., y Da Silva, E. (2017). El perfil emprendedor y la predisposición para innovar de los gestores de las mpe's turísticas. El caso del barrio de Praia Grande en São Luís, Maranhão, Brasil. Estudios y Perspectivas en turismo, 26(1), 107-127. https://www.redalyc.org/pdf/1807/180749182006.pdf

Moreno, A. (2012). Historia del turismo: Una investigación necesaria. Turismo y sostenibilidad: $V$ jornadas de investigación en turismo, 105-126. https://idus.us.es/xmlui/bitstream/handle/11441/53098/morenogarrido.pdf?sequence $=1 \&$ is Allowed $=\mathrm{y}$ 
Moriano, J., Topa, G., Molero, F., Entenaza, A., y Levi, J. (2012). Autoeficacia para el Liderazgo Emprendedor. Adaptación y Validación de la Escala CESE en España. Red de Revistas Científicas de América Latina y el Caribe, España y Portugal, 28, 171-179.

OMT. (21 de enero de 2020). UNWTO. Recuperado de Organización Mundial de Turismo: https://www2.unwto.org/es/content/acerca-de-la-omt

Omar, N. A., Aris, H. M., \& Nazri, M. A. (2016). The effect of entrepreneurial orientation, innovation capability and knowledge creation on firm performance: A perspective on small scale entrepreneurs. Jurnal Pengurusan (UKM Journal of Management), 48.

Oyarvide, H., Nazareno, T., Roldán, A., y Ferrales, Y. (2016). Emprendimiento como factor del desarrollo turístico rural sostenible. Retos de la dirección, 10 (1).

Palacio, A., Arévalo, P., y Guadalupe, J. (2017). Tipología de la Innovación Empresarial según $\begin{array}{llll}\text { Manual de Oslo. } & \text { CienciAmérica, } & \text { 85-90. }\end{array}$ https://dialnet.unirioja.es/servlet/articulo?codigo=6163724

Piñeiro-Chousa, J., López-Cabarcos, M. Á., Romero-Castro, N. M., \& Pérez-Pico, A. M. (2020). Innovation, entrepreneurship and knowledge in the business scientific field: Mapping the research front. Journal of Business Research, 115, 475-485.

Presenza, A., Abbate, T., Meleddu, M., \& Sheehan, L. (2020). Start-up entrepreneurs' personality traits. An exploratory analysis of the Italian tourism industry. Current Issues in Tourism, 23(17), 2146-2164.

Quero, M. (2010). Confiabilidad y Coeficiente Alpha de Cronbach. Telos, 12(2), 248-252. https://www.redalyc.org/pdf/993/99315569010.pdf

Rodriguez-Sanchez, I., Williams, A. M., \& Brotons, M. (2019). The innovation journey of newto-tourism entrepreneurs. Current Issues in Tourism, 22(8), 877-903.

Salvador , C., y Morales, J. (2009). Autoeficacia Emprendedora en Jóvenes Mexicanos. ¿Cómo Afecta la Satisfacción Vital y la Inteligencia Emocional Percibida? Revista Interamericana de Psicología, 43(2), 268-278. https://www.redalyc.org/pdf/284/28412891008.pdf

Sánchez, J. C. (2010). Evaluación de la Personalidad Emprendedora: Validez Factorial del Cuestionario de Orientación. Revista Latinoamericana de Psicología, 42(1), 41-52. https://www.redalyc.org/pdf/805/80515880004.pdf

Sancho, A., Buhalis, D., Gallego, J., Jaume Mata, J., Navarro, S., Osorio, E., Perdro, A., Ramos, S., y Ruiz, P. (2011). Turismo: Concepto y Definiciones. Introducción al Turismo (OMT), 43-57. http://www.utntyh.com/wp-content/uploads/2011/09/INTRODUCCION-ALTURISMO-OMT.pdf

Selznick, B. S., \& Mayhew, M. J. (2018). Measuring undergraduates' innovation capacities. Research in Higher Education, 59(6), 744-764.

Shahab, Y., Chengang, Y., Arbizu, A. D., \& Haider, M. J. (2019). Entrepreneurial self-efficacy and intention: do entrepreneurial creativity and education matter?. International Journal of Entrepreneurial Behavior \& Research. 25(2), 259-280.

Shepherd, D.A., (2015). Party on! A call for entrepreneurship research that is more interactive, activity based, cognitively hot, compassionate, and prosocial. Journal of Business Venturing, 30 (4), 489-507. http://doi.org/10.1016/j.jbusvent.2015.02.001

Solvoll, S., Alsos, G.A., Bulanova, O., (2015). Tourism entrepreneurship-Review and future directions. Scand. Journal of Hospitality and Tourism 15(sup 1), 120-137. 
Tsai, K.H., Chang, H.C. and Peng, C.Y. (2016), "Extending the link between entrepreneurial selfefficacy and intention: a moderated mediation model", International Entrepreneurship and Management Journal, 12(2), 445-463.

Ubierna, F. (2015). La intención emprendedora y el estudiante universitario de turismo: Analisis comparativo de grado y máster. International Journal of Scientific Management and Tourism, 1, 235-273. https://dialnet.unirioja.es/servlet/articulo?codigo=5665867.

Volery, T., Mazzarol, T., (2015). The evolution of the small business and entrepreneurship field: a bibliometric investigation of articles published in the International Small Business Journal. Int. Small Bus. J. 33 (4), 374-396.

Webster, C., Ivanov, S., (2014). Transforming competitiveness into economic benefits: does tourism stimulate economic growth in more competitive destinations? Tourism Management.40, 137-140. 